\title{
Insulin sensitivity is impaired in heterozygous carriers of lipoprotein lipase deficiency
}

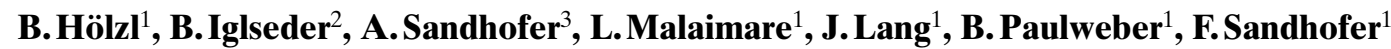 \\ ${ }^{1}$ First Department of Internal Medicine, St. Johanns Spital, Salzburg, Austria \\ ${ }^{2}$ Department of Neurology, Christian Doppler Klinik, Salzburg, Austria \\ ${ }^{3}$ Department of Medicine, University of Innsbruck, Innsbruck, Austria
}

\begin{abstract}
Aims/hypothesis. Several studies have investigated the lipoprotein phenotype in heterozygous carriers of a defective lipoprotein lipase allele. We studied whether heterozygosity for lipoprotein lipase deficiency also affects glucose metabolism beyond its effect on plasma lipids.

Methods. To address this question 85 heterozygous carriers of either a missense mutation (Gly188Glu) or a splice site mutation $(\mathrm{C} \rightarrow \mathrm{A}$ in position -3 at the acceptor splice site of intron 6) in the $L P L$ gene which both result in a catalytically inactive product were compared with 108 unaffected subjects from the same families.

Results. Carriers for one of these mutations had higher fasting insulin levels but only a trend towards increased fasting blood glucose concentrations could be detected. HOMA index values were significantly higher in carriers than in non-carriers. Furthermore, in carriers, a significantly higher BMI and a trend towards higher systolic and diastolic blood pressure were observed. Carriers also had significantly higher fasting triglycerides, lower HDL cholesterol, and li-
\end{abstract}

poprotein lipase particles of smaller size, confirming previous reports. Among carriers, subjects with one rare allele of the SstI polymorphism in the apo CIII gene had significantly higher plasma triglyceride levels than those with two common SstI alleles. This difference could not be observed in non-carriers of a mutant lipoprotein-lipase allele. The mean intima media thickness of the carotid arteries was slightly, but not significantly higher in carriers when compared with non-carriers.

Conclusion/interpretation. This study shows that carrier status of one defective lipoprotein-lipase allele is associated with impaired insulin sensitivity, an atherogenic lipoprotein profile and other characteristics of the metabolic syndrome, which are risk factors for atherosclerotic vascular disease. A higher incidence of atherosclerotic vascular disease, however, could not be firmly established in carriers of this study population. [Diabetologia (2002) 45: 377-384]

Keywords Lipoprotein lipase, gene mutation, lipoproteins, insulin resistance, intima media thickness, atherosclerotic vascular disease.
Received: 24 August 2001 and in revised form: 13 November 2001

Corresponding author: B. Hölzl M. D., First Department of Internal Medicine, St. Johanns Spital, Müllner Hauptstraße 48, 5020 Salzburg, Austria, e-mail: b.hoelzl@lks.at

Abbreviations: LPL, Lipoprotein lipase; Lp(a), lipoprotein(a); apo, apolipoprotein; IDL, intermediate density lipoproteins; HOMA, homeostasis model assessment of insulin resistance; S-BP, mean systolic ambulatory blood pressure; D-BP, mean diastolic ambulatory blood pressure; IMT, intima media thickness; CCA, common carotid artery; ICA, internal carotid artery
Lipoprotein lipase (LPL) hydrolyses triglycerides in triglyceride-rich lipoproteins, thus forming remnant particles which are either degraded by the liver or converted to IDL and LDL. Free cholesterol and phospholipids from the surface of chylomicrons are transferred to HDL. LPL is bound to the capillary endothelium mainly in muscle and adipose tissue and can be released by i.v. injection of heparin. Complete deficiency of this enzyme results in a massive accumulation of chylomicrons in plasma [1]. 
A number of structural mutations in the $L P L$ gene have been described [2,3]. Patients homozygous for mutations in the $L P L$ gene which result in a catalytically inactive enzyme protein suffer from familial hyperchylomicronemia and are easily detected in early infancy by the milky appearance of their fasting plasma and their hepato-splenomegaly. Their plasma triglycerides are greatly increased and their HDL cholesterol is low, while total cholesterol is nearly normal or moderately higher [4]. The frequency of this metabolic defect is about 1 for each million [4]. From this frequency of homozygous patients it can be deduced that in the general population about 1 in 500 is a heterozygous carrier of a defective $L P L$ allele. Carriers of one defective allele cannot be detected by screening of plasma lipid levels. Their $L P L$ activity is reduced to about half the activity of healthy control subjects with a considerable overlap [5]. Therefore, the only way to detect carriers is to screen for mutations in the $L P L$ gene.

Many mutations which do not result in a complete loss of enzymatic activity, but only cause a moderate decrease of lipolytic function have been found in the $L P L$ gene. Some of these mutations are quite common in most populations $[2,6]$ and many studies on the phenotypic expression of mutations in the $L P L$ gene have been done on mutations with only a modest or no effect on lipolytic activity. In heterozygous carriers of some of these mutations an increase of plasma triglycerides and a decrease in HDL cholesterol level, the occurrence of small dense LDL, and an increased risk for atherosclerotic vascular disease have been described but the results are inconsistent or could only be demonstrated in certain subgroups. These studies have been extensively reviewed recently $[2,6,7]$.

A few studies describe the phenotype of heterozygous carriers of mutations in the $L P L$ gene which completely abolish lipolytic function of the gene product. Because LPL plays a major role in the metabolism of triglyceride-rich lipoproteins, these studies focussed on plasma lipids. Investigators studied heterozygous carriers of the Gly188Glu mutation and found a significant increase of plasma triglycerides, VLDL cholesterol, and apolipoprotein B (apo B), and a decrease of HDL- and LDL-cholesterol only in a subgroup of individuals over 40 years old [8]. A similar pattern of plasma lipids has been described in heterozygous carriers from French-Canadian families with the Pro207Leu mutation [9]. In a study of 8 heterozygous carriers of the Gly188Glu mutation and 8 control subjects from two families the carriers had clearly reduced $L P L$ activity, but their fasting plasma triglycerides and HDL cholesterol levels were not significantly different from the values of the non-carriers; the carriers had LDL particles of small size and exhibited pronounced postprandial lipemia in a fat tolerance test [10].
During the past few years, 8 patients with primary hyperchylomicronemia due to a complete or nearly complete $L P L$ deficiency were admitted to our lipid clinic. Altogether 4 of them were homozygous for the Gly188Glu mutation [5], 2 were homozygous for a $\mathrm{C} \rightarrow \mathrm{A}$ mutation in position -3 at the acceptor splice site of intron 6 causing aberrant splicing and inactive LPL [11], and 2 were compound heterozygotes for these two mutations [11]. Because all these patients lived in a clearly defined area near Salzburg, we assumed that a rather large number of heterozygotes could be detected in this region. Therefore, collaborating with local physicians, we screened about 2000 individuals from this region. In the 85 heterozygotes individuals detected for one of these two mutations we investigated various parameters of lipid and glucose metabolism.

\section{Subjects and methods}

Subjects. In a clearly defined geographical area where 8 patients with primary hyperchylomicronemia due to a Gly188Glu missense mutation or a 3' splice site mutation in intron 6 of the $L P L$ gene had been previously detected, about 2000 subjects were screened for the presence of one of these 2 mutations. The methods of screening for the Gly188Glu mutation and for the 3 ' splice site mutation in intron 6 have been described previously $[5,11]$. Heterozygous carriers were detected in 51 families. Subsequently, other members of these families were screened to identify further carriers or non-carriers. A total of 85 carriers (41 with the Gly188Glu mutation and 44 with the 3 ' splice site mutation in intron 6) and 108 noncarriers were found. One carrier and one non-carrier were diabetics. Patients on lipid-lowering or antihypertensive drugs were excluded for statistical analyses of serum lipids or blood pressure, two patients with overt diabetes for analysis of parameters of glucose metabolism. There was no difference in the number of smokers between carriers and non-carriers. All subjects gave their informed consent and the study was carried out in accordance with the Declaration of Helsinki as revised in 1996.

Laboratory analyses. Venous blood was collected after an overnight fast. Fasting insulin was measured using the IMX insulin kit (Abbot, Vienna, Austria). The reduction of plasma glucose after i.v. insulin (k-ITT) was estimated using the short insulin tolerance test as described previously [12]. HOMA indices were calculated using the formula fasting blood glucose $(\mathrm{mg} / \mathrm{dl})$ times fasting insulin $(\mathrm{mU} / \mathrm{l}) / 405$ [17]. Total serum cholesterol, triglycerides, HDL cholesterol, LDL cholesterol, Lp(a), Apo AI and Apo B were determined enzymatically with commercially available kits (Hoffmann-LaRoche, Vienna, Austria). LDL size was determined by gradient gel electrophoresis using a kit from Zaxis (Hudson, Ohio, USA). Typing of the SstI polymorphism near the 3' end of the apo C III gene was performed using a protocol previously described [18].

24-h ambulatory blood pressure measurements. 24-h ambulatory blood pressure measurements were done in 50 carriers and 78 non-carriers using the Diasys-Integra blood pressure monitoring system from Novacor (Rueil-Malmaison, France). None of these subjects was on antihypertensive therapy. 
Table 1. Clinical parameters in non-carriers and carriers of a mutant $L P L$ allele

\begin{tabular}{|c|c|c|c|c|c|c|}
\hline \multirow[b]{2}{*}{ Parameter } & \multicolumn{2}{|c|}{ Non-carriers } & \multicolumn{2}{|c|}{ Carriers } & \multirow[b]{2}{*}{$p$} & \multirow[b]{2}{*}{$\mathrm{p}^{\mathrm{a}}$} \\
\hline & $\bar{n}$ & means $\pm \mathrm{SEM}$ & $\bar{n}$ & means $\pm \mathrm{SEM}$ & & \\
\hline Age (years) & 108 & $40.8 \pm 1.8$ & 85 & $42.5 \pm 2.0$ & n. s. ${ }^{d}$ & n. s. ${ }^{d}$ \\
\hline Body fat (\%) & 18 & $26.3 \pm 2.5$ & 33 & $26.9 \pm 1.8$ & n. s. ${ }^{\mathrm{d}}$ & 0.047 \\
\hline $\mathrm{S}-\mathrm{BP}($ day $)(\mathrm{mmHg})^{\mathrm{b}}$ & 78 & $120.9 \pm 1.6$ & 50 & $124.5 \pm 2.0$ & n. s. ${ }^{d}$ & n. s. ${ }^{\mathrm{d}}$ \\
\hline $\mathrm{D}-\mathrm{BP}($ day $)(\mathrm{mmHg})^{\mathrm{b}}$ & 78 & $76.3 \pm 1.0$ & 50 & $79.5 \pm 1.1$ & 0.043 & 0.076 \\
\hline CCA mean $(\mu \mathrm{m})$ & 82 & $755.9 \pm 30.8$ & 70 & $770.4 \pm 39.2$ & n. s. ${ }^{d}$ & n. s. ${ }^{\mathrm{d}}$ \\
\hline ICA mean $(\mu \mathrm{m})$ & 82 & $775.3 \pm 30.3$ & 70 & $785.0 \pm 37.7$ & n. s. ${ }^{\mathrm{d}}$ & n. s. ${ }^{\mathrm{d}}$ \\
\hline Tot-C $(\mathrm{mg} / \mathrm{dl})^{\mathrm{b}}$ & 105 & $198.3 \pm 4.0$ & 82 & $207.2 \pm 5.4$ & n. s. ${ }^{d}$ & n. s. ${ }^{d}$ \\
\hline LDL-C $(\mathrm{mg} / \mathrm{dl})^{\mathrm{b}}$ & 105 & $124.5 \pm 3.6$ & 82 & $131.9 \pm 4.9$ & n. s. ${ }^{\mathrm{d}}$ & n. s. ${ }^{\mathrm{d}}$ \\
\hline $\mathrm{TG}(\mathrm{mg} / \mathrm{dl})^{\mathrm{b}}$ & 105 & $88.6 \pm 4.6$ & 82 & $142.9 \pm 11.0$ & $<0.0005^{\mathrm{c}}$ & $<0.0005^{\mathrm{c}}$ \\
\hline HDL-C $(\mathrm{mg} / \mathrm{dl})^{\mathrm{b}}$ & 105 & $61.0 \pm 1.8$ & 82 & $50.5 \pm 1.5$ & $<0.0005$ & $<0.0005$ \\
\hline $\mathrm{Lp}(\mathrm{a})(\mathrm{mg} / \mathrm{dl})$ & 99 & $20.4 \pm 2.9$ & 79 & $29.2 \pm 3.8$ & 0.070 & 0.115 \\
\hline Apo AI $(\mathrm{mg} / \mathrm{dl})^{\mathrm{b}}$ & 105 & $150.0 \pm 2.8$ & 82 & $147.7 \pm 3.6$ & n. s. ${ }^{d}$ & n. s. ${ }^{\mathrm{d}}$ \\
\hline Apo B (mg/dl $)^{b}$ & 105 & $108.0 \pm 2.5$ & 82 & $117.6 \pm 3.9$ & 0.032 & 0.102 \\
\hline F-BG (mg/dl) & 106 & $92.1 \pm 1.2$ & 84 & $92.9 \pm 3.0$ & n. s. ${ }^{\mathrm{d}}$ & n. s. ${ }^{\mathrm{d}}$ \\
\hline F-Insulin $(\mu \mathrm{U} / \mathrm{ml})$ & 107 & $7.2 \pm 0.4$ & 79 & $10.1 \pm 0.9$ & 0.001 & 0.008 \\
\hline k-ITT (\%/minute) & 106 & $4.6 \pm 0.1$ & 79 & $4.2 \pm 0.2$ & 0.03 & n. s. ${ }^{d}$ \\
\hline HOMA index & 106 & $1.7 \pm 0.2$ & 79 & $2.3 \pm 0.2$ & $0.006^{\mathrm{c}}$ & $0.03^{\mathrm{c}}$ \\
\hline Fibrinogen $(\mathrm{mg} / \mathrm{dl})$ & 104 & $257.6 \pm 5.1$ & 83 & $282.8 \pm 7.7$ & 0.007 & 0.037 \\
\hline
\end{tabular}

a After adjustment for age, sex, BMI; for BMI adjusted for age $\quad{ }^{\mathrm{c}}$ Calculated from the analysis of log-transformed data and sex only

${ }^{\mathrm{b}}$ Excluding cases on medication

${ }^{\mathrm{d}}$ NS not significant: $p>0.15$

IMT measurement. Intima media thickness of the carotid arteries was measured by high resolution B-mode ultrasound (HDI 3000 CV from ATL, Munich, Germany) according to a protocol of other investigators [19].

Statistics. Mean levels of all tested clinical and biochemical parameters in the cohort of non-carriers were compared to mean levels in the cohort of carriers either using the independent samples $t$ test or after adjustment for age, sex and BMI employing appropriate general linear models. General linear model analysis was also used to examine the possible presence of an interaction effect between the $L P L$ mutations and the common and rare allele of the SstI polymorphism with respect to mean levels of triglycerides.

All analyses were done using the SPSS statistical software, version 10.0 (SPSS, Chicago, Ill., USA). A $p$ value of less than 0.05 was considered significant.

\section{Results}

Altogether 85 heterozygous carriers (43 males and 42 females, 41 carrying the Gly188Glu mutation and 44 with the 3 ' splice site mutation) and 108 non-carriers (53 males and 55 females) were studied. Clinical characteristics and laboratory parameters of carriers and non-carriers are summarized in Table 1.

Glucose metabolism. Carriers showed significantly higher levels of fasting insulin and HOMA index. These differences remained statistically significant after adjustment for age, sex and body mass index. The differences were also significant when the carriers of the Gly188Glu mutation and carriers of the splice site mutation in intron 6 were considered separately (Table 2).

Carriers also showed a trend towards higher fasting glucose concentrations and lower k-ITT values. In carriers of the Gly188Glu mutation the difference in k-ITT was significant (Table 2). Two patients with overt Type II (non-insulin-dependent) diabetes mellitus (one carrier and one non-carrier) were omitted from the calculations.

$B M I$. Carriers had a significantly higher BMI. This difference remained significant after adjustment for age and sex (Table 1).

Blood pressure. Carriers showed a small but not significant increase of systolic and diastolic daytime ambulatory blood pressure (Table 1).

Plasma lipids and lipoproteins. Carriers had higher fasting triglycerides, lower HDL cholesterol, and LDL particles of smaller size (Table 1). All these differences were statistically significant and remained significant after adjustment for age, sex and BMI.

Among carriers, a trend towards increases in $\mathrm{Lp}$ (a) levels was found. No difference between carriers and non-carriers could be demonstrated with regard to total cholesterol, LDL cholesterol, apo AI, and apo B. Carriers also had higher plasma fibrinogen levels.

Sst polymorphism in the apo CIII gene and plasma triglycerides. A possible interaction between the SstI polymorphism in the apo CIII gene and carrier status 
Table 2. Clinical parameters in non-carriers and carriers of the Gly188Glu mutation or the splice site mutation in intron 6 (6ss)

\begin{tabular}{|c|c|c|c|c|c|c|c|c|c|c|}
\hline \multirow[b]{2}{*}{ Parameter } & \multicolumn{2}{|c|}{ Non-carriers } & \multicolumn{2}{|c|}{ Carriers (Gly188Glu) } & \multirow[b]{2}{*}{$p$} & \multirow[b]{2}{*}{$\mathrm{p}^{\mathrm{a}}$} & \multicolumn{2}{|c|}{ Carriers (6 ss) } & \multirow[b]{2}{*}{$p$} & \multirow[b]{2}{*}{$\mathrm{p}^{\mathrm{a}}$} \\
\hline & $\bar{n}$ & $\overline{\text { means } \pm \mathrm{SEM}}$ & $n$ & $\overline{\text { means } \pm \mathrm{SEM}}$ & & & $n$ & means $\pm \mathrm{SEM}$ & & \\
\hline$\overline{\mathrm{BMI}}(\mathrm{kg} / \mathrm{m} 2)$ & 108 & $23.9 \pm 0.4$ & 40 & $26.4 \pm 0.7$ & 0.001 & 0.002 & 43 & $24.8 \pm 0.8$ & n.s. ${ }^{d}$ & n.s. $^{d}$ \\
\hline Body fat (\%) & 18 & $26.3 \pm 2.5$ & 17 & $26.7 \pm 2.8$ & n. $s^{d}$ & 0.031 & 16 & $27.1 \pm 2.4$ & n.s. ${ }^{d}$ & n.s. ${ }^{d}$ \\
\hline $\mathrm{S}-\mathrm{BP}(\mathrm{dav})(\mathrm{mmHg})^{\mathrm{b}}$ & 78 & $120.9 \pm 1.6$ & 23 & $126.1 \pm 3.0$ & 0.11 & n. s. $^{\mathrm{d}}$ & 27 & $122.9 \pm 2.5$ & n. s. $^{\mathrm{d}}$ & n. s. ${ }^{\mathrm{d}}$ \\
\hline $\mathrm{D}-\mathrm{BP}($ day $)(\mathrm{mmHg})^{\mathrm{b}}$ & 78 & $76.3 \pm 1.0$ & 23 & $80.4 \pm 1.6$ & 0.05 & n.s. & 27 & $78.7 \pm 1.4$ & n.s. ${ }^{d}$ & n.s. ${ }^{d}$ \\
\hline CCA mean $(\mu \mathrm{m})$ & 82 & $755.9 \pm 30.8$ & 35 & $774.3 \pm 59.4$ & n. $s^{d}$ & n. $s^{d}$ & 35 & $766.4 \pm 52.1$ & n. $s^{d}$ & n. $s^{d}$ \\
\hline ICA mean $(\mu \mathrm{m})$ & 82 & $775.3 \pm 30.3$ & 35 & $777.5 \pm 56.1$ & n. s. ${ }^{d}$ & n. s. ${ }^{\mathrm{d}}$ & 35 & $792.3 \pm 51.3$ & n. s. ${ }^{d}$ & n. s. ${ }^{\mathrm{d}}$ \\
\hline $\mathrm{TG}(\mathrm{mg} / \mathrm{dl})^{\mathrm{b}}$ & 105 & $88.6 \pm 4.6$ & 41 & $140.6 \pm 14.9$ & $<0.0005^{\mathrm{c}}$ & $<0.0005^{\mathrm{c}}$ & 41 & $145.2 \pm 16.4$ & $<0.0005^{\mathrm{c}}$ & $<0.0005^{\mathrm{c}}$ \\
\hline $\mathrm{HDL}-\mathrm{C}(\mathrm{mg} / \mathrm{dl})^{\mathrm{b}}$ & 105 & $61.0 \pm 1.8$ & 41 & $50.7 \pm 2.3$ & 0.001 & $<0.016$ & 41 & $50.3 \pm 1.9$ & 0.0008 & 0.0011 \\
\hline LDL size ${ }^{b}$ & 83 & $267.2 \pm 1.1$ & 22 & $257.2 \pm 1.5$ & $<0.0005$ & $<0.0005$ & 21 & $257.6 \pm 2.6$ & $<0.0005$ & 0.0005 \\
\hline F-BG $(\mathrm{mg} / \mathrm{dl})$ & 107 & $92.6 \pm 1.2$ & 40 & $94.3 \pm 3.3$ & n. s. ${ }^{d}$ & n. s. ${ }^{d}$ & 44 & $91.7 \pm 1.9$ & n. s. ${ }^{d}$ & n. s. ${ }^{d}$ \\
\hline F-Insulin $(\mu \mathrm{U} / \mathrm{ml})$ & 108 & $7.2 \pm 0.4$ & 37 & $10.1 \pm 1.3$ & 0.004 & 0.024 & 42 & $10.1 \pm 1.3$ & 0.004 & 0.012 \\
\hline k-ITT & 107 & $4.6 \pm 0.1$ & 37 & $4.1 \pm 0.2$ & 0.022 & 0.13 & 42 & $4.3 \pm 0.2$ & $\mathrm{n} \cdot \mathrm{s}^{\mathrm{d}}$ & $\mathrm{n} \cdot \mathrm{s}^{\mathrm{d}}$ \\
\hline HOMA index & 107 & $1.7 \pm 0.2$ & 37 & $2.4 \pm 0.4$ & $0.014^{\mathrm{c}}$ & $0.048^{c}$ & 42 & $2.3 \pm 0.3$ & $0.016^{\mathrm{c}}$ & $0.031^{\mathrm{c}}$ \\
\hline Fibrinogen $(\mathrm{mg} / \mathrm{dl})$ & 104 & $257.6 \pm 5.1$ & 41 & $279.2 \pm 8.2$ & 0.03 & 0.096 & 42 & $286.3 \pm 13.0$ & 0.014 & 0.027 \\
\hline
\end{tabular}

${ }^{a}$ After adjustment for age, sex, BMI; for BMI adjusted for age $\quad{ }^{\mathrm{c}}$ Calculated from the analysis of log-transformed data and sex only

${ }^{\mathrm{b}}$ Excluding cases on medication

${ }^{\mathrm{d}}$ NS not significant: $p>0.15$

Table 3. Interaction between SstI polymorphism in the $C I I I$ gene and $L P L$ carrier status with respect to serum triglyceride levels

\begin{tabular}{|c|c|c|c|c|c|c|}
\hline & \multicolumn{4}{|c|}{ Triglycerides $(\mathrm{mg} / \mathrm{dl})$ Means \pm SEM } & \multirow[t]{2}{*}{$p$} & \multirow[t]{2}{*}{$p^{\mathrm{a}}$} \\
\hline & $\bar{n}$ & Non-carriers & $n$ & Carriers & & \\
\hline Sst 1 & 91 & $88.2 \pm 5.1$ & 73 & $134.4 \pm 10.2$ & $<0.0005$ & $<0.0005$ \\
\hline Sst 2 & 13 & $90.0 \pm 9.4$ & 8 & $226.5 \pm 59.6$ & 0.003 & 0.021 \\
\hline$p$ & & n. s. ${ }^{b}$ & & 0.024 & & \\
\hline$p^{\mathrm{a}}$ & & n. s. ${ }^{b}$ & & 0.008 & & \\
\hline
\end{tabular}

$p$ values were calculated on log transformed data; Sst 1 - homozygous for the common allele, Sst 2 - heterozygous for the rare allele of the SstI polymorphism. $p$ values for interaction effect between $L P L$ and SstI carrier-status employing a gener-

of an inactive $L P L$ allele with regard to plasma triglyceride levels was studied in 81 carriers and 104 non-carriers. Altogether 73 carriers and 91 non-carriers had two common alleles, 8 carriers and 13 noncarriers had one rare allele of the SstI polymorphism. Among non-carriers, no difference in the concentration of plasma triglycerides could be detected between subjects homozygous for the common allele or heterozygous carriers of the rare allele of the SstI polymorphism. Among carriers, however, subjects carrying one rare SstI allele had significantly higher plasma triglyceride concentrations than subjects with two common SstI alleles (Table 3). Employing a general linear model, the interaction effect between SstI- and LPL carrier status with regard to plasma triglyceride levels was nearly significant $(p=0.089)$ and remained so $(p=0.096)$ after adjustment for sex, age and BMI (Table 3).

Atherosclerotic vascular disease. Mean IMT values of the carotid arteries (common and internal carotid artery) were slightly higher in carriers than in non-carriers (Table 1$)$. Among carriers, 4 individuals (2 males and 2 females), and in the group of non-carriers al linear model: $p=0.089$ without adjustment; $p=0.096$ after adjustment for age, sex, and BMI

${ }^{a}$ After adjustment for age, sex and BMI

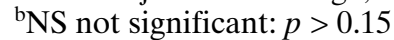

2 males, had definite coronary artery disease (history of myocardial infarction or coronary angioplasty).

\section{Discussion}

The subjects of this study were recruited from a clearly defined geographical area (a valley in the Austrian mountains south of Salzburg where the population mobility always was very low), where eight patients with primary hyperchylomicronemia had previously been detected. These patients were homozygous or compound heterozygous for a defective $L P L$ allele due to a Gly188Glu missense mutation or a splice site mutation in the acceptor splice site of intron 6. Familial hyperchylomicronemia is a rare metabolic disorder with a frequency of 1 in a million. Because the number of patients found in this area is much higher than expected, a relatively large number of heterozygotes for one of these two mutations could be detected by screening the general population of this region. The 85 carriers and 108 non-carriers of this study are members of 51 families. Although it is not possible to clearly define the pedigrees of these 
families a genetic relationship can be assumed. The high frequency of the two mutations is possibly due to founder effects, but this could not be firmly established.

In this study, significantly higher levels of fasting insulin but only a trend towards increased fasting blood glucose values has been found in heterozygous carriers of one mutant allele of the $L P L$ gene, either the missense mutation Gly188Glu or the splice site mutation in intron 6. In carriers, HOMA values were significantly higher than in non-carriers. This finding suggests that insulin sensitivity is reduced in the presence of one defective $L P L$ allele. Carriers also showed an increased BMI, a trend towards a higher blood pressure and increased plasma fibrinogen levels. Together with higher plasma triglycerides, low HDL cholesterol and small dense LDL, all these findings are the characteristic symptoms of the so-called metabolic syndrome. Whereas the alterations of plasma lipoproteins can be explained by reduced lipolytic activity due to one defective $L P L$ allele, the effect on other components of the metabolic syndrome are difficult to interpret. The metabolic syndrome is the consequence of insulin resistance. In our study, carriers obviously showed characteristic findings of insulin resistance and had an increased BMI. Two possible mechanisms for these findings are: the carrier status itself is responsible for insulin resistance, or the carrier status primarily results in an increased BMI which is the cause for insulin resistance or both. When BMI, the mutated $L P L$ allele, age and sex were taken into consideration as factors influencing insulin sensitivity, these four factors explained approximately $15 \%$ of the total variation of fasting insulin and HOMA. The effect of the $L P L$ mutations was of the same magnitude as the effect of BMI, whereas the effect of age and sex was much weaker. Therefore, we suggest that in carriers the mutated $L P L$ allele impaired insulin sensitivity not only by increasing BMI but also by another mechanism independent from BMI.

In our study, carriers showed a highly significant elevation of fasting triglycerides, a decrease in HDL cholesterol, and a smaller size of LDL particles. Due to the low frequency of familial hyperchylomicronemia only a few studies have been done describing the phenotype in heterozygous carriers of mutations resulting in inactive gene products. Our results concerning plasma lipids are consistent with findings reported by other investigators $[8,9,16-18]$. The authors of these studies reported that in heterozygous carriers of the Gly188Glu or Pro207Leu mutation expression of hypertriglyceridaemia is dependent on age, obesity and diabetes [8, 9, 22]. In our study, carriers had significantly higher levels of plasma triglycerides, even after adjustment for age, sex and BMI.

Different mutations of the $L P L$ gene could have different effects on phenotypic expression. In the case of the Gly188Glu mutation, a single amino acid substitution results in complete loss of lipolytic activity [19]. Heterozygotes with this mutation have about half normal lipolytic activity [5]. The splice site mutation in intron 6 results in aberrant splicing: The major transcript shows deletion of exons 6 through 9 and amounts to only about $3 \%$ of the normal transcript in a healthy subject; only trace amounts of a normally spliced transcript can be detected [11]. In homozygotes of the splice site mutation post-heparin $L P L$ activity is extremely low, heterozygotes had variable $L P L$ activities ranging from less than half normal to normal [11]. The effects on carbohydrate and lipid metabolism were observed for both the missense and the splice site mutation. Because these two mutations result in structurally very different gene products, we suggest that the observed metabolic effects are due the reduced $L P L$ activity rather than to a specific alteration of the protein structure.

In various populations, an association of the rare allele of the SstI polymorphism at the 3' end of the apo CIII gene with higher levels of plasma triglycerides has been described $[5,12,13]$. Therefore, we investigated a possible interaction between this polymorphism and plasma triglyceride levels in carriers. In our study, among non-carriers of a mutant $L P L$ gene, subjects with two common SstI alleles had the same triglyceride level as subjects carrying one rare SstI allele. On the other hand among carriers of a mutant $L P L$ allele, individuals with one rare SstI allele had mean serum triglyceride levels nearly twice as high as those found in subjects carrying two common SstI alleles. The calculation of the interaction with a general linear model correcting for age sex, and BMI resulted in a $p$ value of 0.096 . The small number of individuals carrying both a rare $L P L$ mutation and a rare polymorphic allele could make it impossible to prove an interaction. Therefore, we can only suggest that in carriers of one defective $L P L$ allele the SstI polymorphism could possibly modulate the expression of hypertriglyceridemia. Because the SstI polymorphic site is located in the non-coding region of the Apo CIII gene, the effect of the rare SstI allele might be due to a linkage disequilibrium with other functional sequences in or near the apo CIII gene. A linkage disequilibrium of the SstI polymorphism with a variant form of the human apo CIII promoter has been postulated [23]. Whereas the wild type promoter is down-regulated by insulin, the variant promoter is defective in its response to insulin [24]. This might play a role in the case of an additional lipolytic defect. It has been shown that Apo CIII inhibits the lipolytic activity of $L P L$ [25] as well as the uptake of chylomicron remnants by the liver [26].

Thus, the results of this study strongly suggest that carriers of one mutant $L P L$ allele resulting in a lipolytically deficient protein are characterized by insulin resistance, higher triglyceride levels, decreased HDL 
cholesterol, and a predominance of small dense LDL, and other characteristics of a metabolic syndrome which are risk factors for atherosclerotic vascular disease. The question whether heterozygosity for $L P L$ deficiency represents a risk factor for this disease has been addressed in several studies. In a recent review [7] the Mantel-Haenszel stratified analysis of previous reports was used to calculate the summary odds ratio for an association between carrier status of various mutations in the $L P L$ gene and cardiovascular disease. The results of these calculations are inconsistent. Investigators [27] identified 6 subjects with the Gly188Glu mutation among 9259 individuals in a random sample of the Danish population (Copenhagen City Heart Study), 3 heterozygous carriers of this mutation were found among the 948 patients with coronary heart disease. In the present study, we measured IMT of the common and internal carotid arteries. A moderate trend towards higher values was found in carriers but the difference between carriers and noncarriers was not significant. In the group of carriers, 4 subjects and in the group of non-carriers 2 subjects had a history of a coronary event. These findings seem to indicate that the carrier status of one defective $L P L$ allele without any additional cardiovascular risk factor is not associated with a substantially increased risk of atherosclerotic vascular disease, at least in this cohort of carriers. Possibly the mean age of the subjects was too low to detect a difference in IMT between carriers and non-carriers. A long-term clinical follow up of the study cohort will give a more definite answer to this question.

Acknowledgements. The technical assistance of C. Talman, C. Prudl, J. Arrer and C. Garstenauer, is gratefully acknowledged. This study was made possible by the help of the local general practitioners: Dr. K. Stölzl, Dr. V. Schernthaner, Dr. V. Schwab, and Dr. M. Krimplstätter. This work was supported by a grant of the Salzburg Medical Research Society (Medizinische Forschungsgesellschaft Salzburg).

\section{References}

1. Nilsson-Ehle P, Garfinkel AS, Schotz MC (1980) Lipolytic enzymes and plasma lipoprotein metabolism. Annu Rev Biochem 49: 667-693

2. Fisher RM, Humphries SE, Talmud PJ (1997) Common variation in the lipoprotein lipase gene: effects on plasma lipids and risk of atherosclerosis. Atherosclerosis 135: $145-159$

3. Antikainen M, Tenkanen H, Taskinen MR, Ehnholm C (1996) Mutations affecting human lipoprotein lipase gene. Z Gastroenterol 34 [Suppl 3]: 59-61

4. Brunzell JD (1995) Familial lipoprotein lipase deficiency and other causes of the chylomicronemia syndrome. In: Scriver CR, Beaudet AL, Sly WS, Valle D (eds) The Metabolic and Molecular Bases of Inherited Disease. Vol. 2, McCraw-Hill, New York, pp 1913-1932

5. Paulweber B, Wiebusch H, Miesenboeck G et al. (1991) Molecular basis of lipoprotein lipase deficiency in two Aus- trian families with type I hyperlipoproteinemia. Atherosclerosis 86: 239-250

6. Gehrisch S (1999) Common Mutations of the Lipoprotein Lipase Gene and their Clinical Significance. Curr Atheroscler Rep 1: 70-78

7. Hokanson JE (1999) Functional variants in the lipoprotein lipase gene and risk cardiovascular disease. Curr Opin Lipidol 10: 393-399

8. Wilson DE, Emi M, Iverius PH et al. (1990). Phenotypic expression of heterozygous lipoprotein lipase deficiency in the extended pedigree of a proband homozygous for a missense mutation. J Clin Invest 86: 735-750

9. Julien P, Gagne PC, Murthy MR et al. (1998) Dyslipidemias associated with heterozygous lipoprotein lipase mutations in the French-Canadian population. Hum Mutat [Suppl 1]: S148-S153

10. Miesenbock G, Holzl B, Foger B et al. (1993) Heterozygous lipoprotein lipase deficiency due to a missense mutation as the cause of impaired triglyceride tolerance with multiple lipoprotein abnormalities. J Clin Invest 91: 448-455

11. Holzl B, Huber R, Paulweber B, Patsch JR, Sandhofer F (1994). Lipoprotein lipase deficiency due to a 3' splice site mutation in intron 6 of the lipoprotein lipase gene. J Lipid Res 35: 2161-2169

12. Holzl B, Kraft HG, Wiebusch H, Sandhofer A et al. (2000) Two novel mutations in the lipoprotein lipase gene in a family with marked hypertriglyceridemia in heterozygous carriers. Potential interaction with the polymorphic marker D1S104 on chromosome 1q21-q23. J Lipid Res 41: 734-741

13. Matthews DR, Hosker JP, Rudenski AS, Naylor BA, Treacher DF, Turner RC (1985) Homeostasis model assessment: insulin resistance and beta-cell function from fasting plasma glucose and insulin concentrations in man. Diabetologia 28: 412-419

14. Dallinga-Thie GM, Bu XD, Linde-Sibenius TM, Rotter JI, Lusis AJ, De Bruin TW (1996) Apolipoprotein A-I/C-III/ A-IV gene cluster in familial combined hyperlipidemia: effects on LDL-cholesterol and apolipoproteins B and C- III. J Lipid Res 37: 136-147

15. The ACAPS Group (1992) Rationale and design for the Asymptomatic Carotid Artery Plaque Study (ACAPS). Control Clin Trials 13: 293-314

16. Bijvoet S, Gagne SE, Moorjani S et al. (1996) Alterations in plasma lipoproteins and apolipoproteins before the age of 40 in heterozygotes for lipoprotein lipase deficiency. J Lipid Res 37: 640-650

17. Julien P, Vohl MC, Gaudet D et al. (1997) Hyperinsulinemia and abdominal obesity affect the expression of hypertriglyceridemia in heterozygous familial lipoprotein lipase deficiency. Diabetes 46: 2063-2068

18. Hokanson JE, Brunzell JD, Jarvik GP, Wijsman EM, Austin MA (1999) Linkage of low-density lipoprotein size to the lipoprotein lipase gene in heterozygous lipoprotein lipase deficiency. Am J Hum Genet 64: 608-618

19. Emi M, Wilson DE, Iverius PH et al. (1990) Missense mutation (Gly-Glu188) of human lipoprotein lipase imparting functional deficiency. J Biol Chem 265: 5910-5916

20. Kessling AM, Horsthemke B, Humphries SE (1985) A study of DNA polymorphisms around the human apolipoprotein AI gene in hyperlipidaemic and normal individuals. Clin Genet 28: 296-306

21. Rees A, Stocks J, Sharpe CR, Vella MA et al. (1985). Deoxyribonucleic acid polymorphism in the apolipoprotein A-1-C-III gene cluster. Association with hypertriglyceridemia. J Clin Invest 76: 1090-1095 
22. Paulweber B, Friedl W, Krempler F, Humphries SE, Sandhofer F (1988) Genetic variation in the apolipoprotein AICIII-AIV gene cluster and coronary heart disease. Atherosclerosis 73: 125-133

23. Surguchov AP, Page GP, Smith L, Patsch W, Boerwinkle E (1996) Polymorphic markers in apolipoprotein C-III gene flanking regions and hypertriglyceridemia. Arterioscler Thromb Vasc Biol 16: 941-947

24. Li WW, Dammerman MM, Smith JD, Metzger S, Breslow JL, Leff T (1995) Common genetic variation in the promoter of the human apo CIII gene abolishes regulation by insulin and may contribute to hypertriglyceridemia. J Clin Invest 96: 2601-2605
25. McConathy WJ, Gesquiere JC, Bass H, Tartar A, Fruchart JC, Wang CS (1992) Inhibition of lipoprotein lipase activity by synthetic peptides of apolipoprotein C-III. J Lipid Res 33: $995-1003$

26. Clavey V, Lestavel-Delattre S, Copin C, Bard JM, Fruchart JC (1995) Modulation of lipoprotein B binding to the LDL receptor by exogenous lipids and apolipoproteins CI, CII, CIII, and E. Arterioscler Thromb Vasc Biol 15: 963-971

27. Nordestgaard BG, Abildgaard S, Wittrup HH, Steffensen R, Jensen G, Tybjaerg-Hansen A (1997) Heterozygous lipoprotein lipase deficiency: frequency in the general population, effect on plasma lipid levels, and risk of ischemic heart disease. Circulation 96: 1737-1744 